\title{
STOCHASTIC PROCESSES AS FOURIER INTEGRALS AND DILATION OF VECTOR MEASURES
}

\section{CHRISTIAN HOUDRÉ}

1. Introduction. In this note we give an overview of some recent advances in representations of stochastic processes as Fourier integrals. These advances provide a Plancherel and a Hausdorff-Young theory for stochastic processes and random measures which were not previously available. We expect several applications of these methods, two of which are: existence results for linear stochastic differential equations (see Theorem 6) and a framework in which to develop a Fourier theory for the ubiquitous white noise model.

The idea of representing a stochastic process as a Fourier series or integral goes back at least to the work of Slutsky [S] and Cramér [C], where such representations were derived for mean squared continuous stationary processes. Without the stationarity assumption, and via vector measures, Phillips [Ph] and Kluvánek [K] also obtained Fourier representation theorems for (strongly) continuous processes. Typical assumptions made to derive these Fourier integrals are the continuity of the process, the global norm boundedness of the representing vector measure, as well as its $\sigma$ additivity, or the orthogonality of its increments. However, for basic classes of stochastic processes these assumptions are not verified, e.g., a process with orthogonal increments is not necessarily continuous, while a white measure is only locally bounded. In [Ho2], the above restrictions are relaxed. The presentation of the approach developed therein unfolds as follows: we first replace convergence by summability and obtain a representation theorem for the corresponding continuous processes. We then also replace continuity by measurability, and present a Plancherel and a Hausdorff-Young type theorem for random measures (Theorems 2 and 3). We then show that these random measures can be dilated to orthogonally scattered ones. For this dilation result the heart of our method is Theorem 4, which is a Grothendieck type inequality. Finally, a study of a class of linear stochastic differential equations is presented.

2. Some preliminaries. Let $(\Omega, \mathscr{B}, \mathscr{P})$ be a probability space and for $1 \leq \alpha \leq 2$, let $L^{\alpha}(\Omega, \mathscr{B}, \mathscr{P})\left(L^{\alpha}(\mathscr{P})\right.$ for short $)$ be the space of random variables with finite $\alpha$ th moment (for probabilistic considerations the case $\alpha>2$ is of little interest, furthermore included in $\alpha=2)$. On $L^{\alpha}(\mathscr{P})$ the norm is denoted by $\|\cdot\|_{\alpha}$ and for $Y$ and $Z$ in $L^{\alpha}(\mathscr{P})$, the "inner product"

Received by the editors January 31, 1989.

1980 Mathematics Subject Classification (1985 Revision). Primary 60G12; Secondary $46 \mathrm{G} 10$. 0227.

Supported by the AFOSR Contract No. F49620 85 C 0144 and ONR No. N00014 86 C 
of $Y$ and $Z$ is $\langle Y, Z\rangle_{\alpha}=\mathscr{E} Y Z^{\langle\alpha-1\rangle}$, where $Z^{\langle\alpha\rangle}=|Z|^{\alpha-1} \bar{Z}$ and $\mathscr{E}$ is the expectation. For $1 \leq \beta \leq+\infty, L^{\beta}(\mathbf{R})$ is the usual Lebesgue space with associated norm denoted by $\|\cdot\|_{\beta}$. We also denote by $C_{0}(\mathbf{R})\left(\right.$ resp. $\left.C_{c}(\mathbf{R})\right)$ the space of continuous functions vanishing at infinity (resp. with compact support) and the uniform norm by $\|\cdot\|_{\infty}$. Finally, $L^{\beta}(\mathbf{R})^{V}$ is the space of functions $f \in L^{1}(\mathbf{R})$ and such that $\hat{f} \in L^{\beta}(\mathbf{R})$. Let $\mathscr{B}(\mathbf{R})$ be the Borel $\sigma$-ring of $\mathbf{R}$ and let $\mathscr{B}_{0}(\mathbf{R})$ be the $\delta$-ring of elements of $\mathscr{B}(\mathbf{R})$ with finite Lebesgue measure. A (random) measure is a finitely additive set function $\mu: \mathscr{B}_{0}(\mathbf{R}) \rightarrow L^{0}(\mathscr{P})\left(L^{0}(\mathscr{P})\right.$ is the vector space of random variables).

DEFINITION 1. A random measure $\mu$ has finite $\beta$-variation with respect to the $L^{\alpha}$-norm if $\mu$ is $L^{\alpha}(\mathscr{P})$-valued and if $\|\mu\|=\sup \left\{\|\mu\|(A), A \in \mathscr{B}_{0}(\mathbf{R})\right\}$ is finite, where $\|\mu\|(A)=\sup \left\{\left\|\sum_{i=1}^{n} a_{i} \mu\left(A_{i}\right)\right\|_{\alpha}:\left\{A_{i}\right\} \subset \mathscr{B}_{0}(\mathbf{R})\right.$ finite partition of $\left.A, a_{i} \in \mathbf{C},\left\|\sum_{i=1}^{n} a_{i} \chi_{A_{i}}\right\|_{\beta} \leq 1\right\}$.

For $\beta=+\infty$, our definition reduces to the ordinary semivariation but for $1 \leq \beta<+\infty$, it differs from the usual $\beta$-semivariation (see [D]) in that it involves $\beta$ and not its conjugate exponent.

The integration with respect to $\mu$ can now be defined as follows: for a simple function $f: \mathbf{R} \rightarrow \mathbf{C}, f=\sum_{i=1}^{n} a_{i} \chi_{A_{i}}, A_{i} \in \mathscr{B}_{0}(\mathbf{R})$, as usual, the integral is $\int_{\mathbf{R}} f d \mu=\sum_{i=1}^{n} a_{i} \mu\left(A_{i}\right)$. If $\mu$ has finite $\beta$-variation (with respect to the $L^{\alpha}$-norm), then $\left\|\int_{\mathbf{R}} f d \mu\right\|_{\alpha} \leq\|\mu\|(A)\|f\|_{\beta}$, where $A=\bigcup_{i=1}^{n} A_{i}$, hence the integral can be extended to $L^{\beta}(\mathbf{R})$. This is done so, an integration with respect to $\mu$ is always taken in this sense.

Finally, a random measure is orthogonally scattered if $\langle\mu(A), \mu(B)\rangle_{2}=0$ whenever $A \cap B=\varnothing$, and stationarily scattered if

$$
\langle\mu(A), \mu(B)\rangle_{2}=\langle\mu(A+t), \mu(B+t)\rangle_{2},
$$

for $A, B \in \mathscr{B}_{0}(\mathbf{R}), t \in \mathbf{R}$, where $A+t=\{a+t: a \in A\}$. A fundamental example of such a measure is a white measure $W$, i.e., $\langle W(A), W(B)\rangle_{2}=$ $|A \cap B|$, the Lebesgue measure of $A \cap B$. The 2-variation of a white measure is finite but for any $\beta \neq 2$, its $\beta$-variation is infinite.

In the rest of this paper, by a process we always mean a strongly measurable bounded function $X: \mathbf{R} \rightarrow L^{\alpha}(\mathscr{P})$, for some $1 \leq \alpha \leq 2$. With this convention we set the following definition in which the first integral is a Lebesgue-Bochner integral.

Definition 2. A process $X$ is $\beta$-bounded if there exist a constant $K>0$ such that

$$
\left\|\int_{\mathbf{R}} f(t) X_{t} d t\right\|_{\alpha} \leq K\left(\int_{\mathbf{R}}|\hat{f}(t)|^{\beta} d t\right)^{1 / \beta}
$$

for all $f$ in $L^{\beta}(\mathbf{R})^{V}$.

Our definition is clearly motivated by the scalar theory, but also by a $V$-boundedness concept introduced by Bochner [B], which corresponds to the case $\beta=+\infty$. In fact, Bochner also introduced, for $\beta<+\infty$, a $\beta$ boundedness notion (which has not been further studied), wherein $\hat{f}$ above is replaced by $f$. These two definitions will be shown to be dual of one another. 
3. Fourier integrals. Our first result is a duality theorem between random processes and measures. Its proof, goes along classical lines: a representation theorem and a vector valued approximation.

THEOREM 1. A process $X$ is (strongly) continuous and $\beta$-bounded if and only if there exists a (unique) measure $\mu$ of finite $\beta$-variation such that for all $t$,

$$
X_{t}=\lim _{\lambda \rightarrow+\infty} \int_{-\lambda}^{\lambda}\left(1-\frac{|\xi|}{\lambda}\right) e^{i t \xi} d \mu(\xi)
$$

in $L^{\alpha}(\mathscr{P})$, uniformly on compact subsets of $\mathbf{R}$.

When $\mu$ is of bounded semivariation the bounded Borel functions are $\mu$-integrable, hence the above limit is just $\int_{\mathbf{R}} e^{i t \xi} d \mu(\xi)$, and we recover the result of Phillips and Kluvánek. Although it is a characterization, Theorem 1 is highly unsatisfactory because of the continuity requirement on $X$. Even under "weaker" conditions, the best possible results will guarantee convergence only for almost all $t$. For example if $X \in L^{p}(\mathbf{R}, \alpha), 1 \leq p \leq$ $+\infty$, the Lebesgue-Bochner spaces of $L^{\alpha}(\mathscr{P})$-valued functions, the above Cesàro integrals converge in $L^{p}(\mathbf{R}, \alpha)$. this is again unsatisfactory, since for no $t$, does $\int_{-\lambda}^{\lambda}\left(1-\frac{|\xi|}{\lambda}\right) e^{i t \xi} d W(\xi)$ converge, either in $L^{2}(\mathscr{P})$ or in $L^{p}(\mathbf{R}, 2)$, $1 \leq p \leq+\infty$. Theorem 1 does not provide a way of defining the Fourier transform of a white measure.

For some fixed $\alpha$, let $\mathscr{M}^{\beta}$ be the Banach spaces (under the $\beta$-variation norm) of measures with finite $\beta$-variation. Again, for $\mu$ of bounded semivariation, i.e., $\mu \in \mathscr{M}^{\infty}$, the exponentials are $\mu$-integrable, hence $\hat{\mu}=\{\hat{\mu}(t)\}$ makes perfect sense and is an $\infty$-bounded process. In order to define the Fourier transform beyond the space $\mathscr{M}^{\infty}$, our strategy is to think of $\hat{\mu}$ not as a stochastic process, but more globally as a stochastic measure, e.g., for $\mu \in \mathscr{M}^{\infty}, d \hat{\mu}(t)=\hat{\mu}(t) d t \in \mathscr{M}^{1}$. We first tackle the case $\beta=2$, the relevant result being a Plancherel type theorem.

THEOREM 2. There exists a unique linear operator $\Lambda$ from $\mathscr{M}^{2}$ onto itself such that

(i) $\Lambda \mu=\hat{\mu}$, for $\mu \in \mathscr{M}^{2} \cap \mathscr{M}^{\infty}$,

(ii) $\|\Lambda \mu\|=\|\mu\|$.

For $\mu \in \mathscr{M}^{2}$, the unique element $\Lambda \mu \in \mathscr{M}^{2}$ is obviously denoted by $\hat{\mu}$. If $\mu \in \mathscr{M}^{2}$, and if, as in Theorem 1, its Cesàro averages converge, the two definitions of the Fourier transform agree and we have,

$$
d \Lambda \mu(t)=\left(\lim _{\lambda \rightarrow+\infty} \int_{-\lambda}^{\lambda}\left(1-\frac{|\xi|}{\lambda}\right) e^{i t \xi} d \mu(\xi)\right) d t .
$$

It is not difficult to see that $\mu$ (resp. $\hat{\mu}$ ) is orthogonally scattered if and only if $\hat{\mu}$ (resp. $\mu$ ) is stationarily scattered. Since $W$ enjoys both properties so does $\Lambda W=\hat{W}$, moreover $\hat{W}$ is Gaussian if and only if $W$ is also Gaussian, i.e., for each $A \in \mathscr{B}_{0}(\mathbf{R}), W(A)$ is a Gaussian random variable. Finally, $\hat{W}$ can also be recovered from $W$ by the inversion formula: $\hat{W}(A)=W(\hat{A})=$ $\int_{\mathbf{R}} \hat{\chi}_{A} d W$. 
As in the scalar case, for $\beta \neq 2$, a dichotomy occurs. First, for $2<\beta<$ $\infty$, we can interpolate between $\mathscr{M}^{2}$ and $\mathscr{M}^{\infty}$ and thus define the Fourier transform by extension.

THEOREM 3. For $2<\beta<\infty$, the Fourier transform operator is a contraction from $\mathscr{M}^{\beta}$ into $\mathscr{M}^{\gamma}, 1 / \beta+1 / \gamma=1$.

For $2<\beta<\infty$, the Fourier transform shares properties similar to the Fourier transform on $\mathscr{M}^{2}$. In particular, an inversion formula holds and, again, under the conditions of Theorem 1, the two definitions of transform coincide.

For $1 \leq \beta<2$, the approach presented here does not work, and this is not surprising in view of the scalar case. Only the methods developed by Gel'fand (see [GV]) or by Hida (see [Hi]), i.e., generalized processes and random distributions, seem to provide a reasonable way of defining the Fourier transform.

4. Dilations. In this section, we analyze the role of the subclasses of orthogonally scattered measures in the spaces $\mathscr{M}^{\beta}$. Our first result provides a characterization of the elements of $\mathscr{M}^{\beta}$ among the finitely additive set functions from $\mathscr{B}_{0}(\mathbf{R})$ to $L^{\alpha}(\mathscr{P})$.

Theorem 4. Let $1 \leq \alpha \leq 2 \leq \beta<+\infty$ (resp. let $\beta=+\infty)$. A measure $\mu$ has finite $\beta$-variation if and only if there exists a nonnegative function $h$ in $L^{\beta / \beta-2}(\mathbf{R})$ (resp. a finite positive Borel measure $h$ ) such that $\left\|\int_{\mathbf{R}} f d \mu\right\|_{\alpha} \leq$ $\left(\int_{\mathbf{R}}|f|^{2} h d t\right)^{1 / 2}\left(\right.$ resp. $\left.\left\|\int_{\mathbf{R}} f d \mu\right\|_{\alpha} \leq\left(\int_{\mathbf{R}}|f|^{2} d h\right)^{1 / 2}\right)$, for all $f$ in $C_{c}(\mathbf{R})$.

For $\alpha=2$ and $\mu \in \mathscr{M}^{\infty},(f, g) \rightarrow \mathscr{E} \int_{\mathbf{R}} f(t) d \mu(t) \int_{\mathbf{R}} g(t) d \mu(t)$ defines a bounded bilinear functional on $C_{0}(\mathbf{R}) \times C_{0}(\mathbf{R})$, and the existence part in Theorem 4 is just one of the various forms of Grothendieck's inequality (see [Pi]). In general, $h$ above does not have compact support. A case at hand is a white measure for which the above inequality (in the 2-bounded case) becomes equality with $h=1$. Hence, in contrast to the classical Grothendieck inequality a "dominating" measure is not necessarily finite.

As important consequences of Theorem 4 we state two results which for $\alpha=2$ and $\beta=+\infty$ are respectively due to Niemi [N] and Bochner [B]. We state our first result only for $\alpha<2$, the quadratic case with $2 \leq \beta<\infty$ being essentially contained in [Ho1].

Theorem 5. A measure $\mu: \mathscr{B}_{0}(\mathbf{R}) \rightarrow L^{\alpha}(\mathscr{P})$ has finite $\beta$-variation if and only if there exist a probability space $(\tilde{\Omega}, \tilde{\mathscr{B}}, \tilde{\mathscr{P}})$ with $L^{2}(\mathscr{P}) \subset L^{2}(\tilde{\mathscr{P}})$ and a orthogonally scattered, $L^{2}(\tilde{\mathscr{P}})$-valued, measure $\nu$ of finite $(\gamma, \beta)$-variation and $\lambda$ in $L^{2 \alpha / 2-\alpha}(\mathscr{P})$ such that $\mu=\lambda P \nu$, i.e., $\mu(A)=\lambda P \nu(A), A \in \mathscr{B}_{0}(\mathbf{R})$, where $P$ is the orthogonal projection from $L^{2}(\tilde{\mathscr{P}})$ to $L^{2}(\mathscr{P})$.

The methods developed here are useful in finding existence theorems and solutions for linear stochastic differential equations and more generally, for linear stochastic analysis. As an example, we state a last theorem and refer the reader to [Ho2] where more details, in particular, applications to symmetric $\alpha$ stable measures, can be found. 
Theorem 6. Let $Y$ be a continuous $\beta$-bounded process whose spectral measure is orthogonally scattered, and let

$$
L=\sum_{k=0}^{n} \alpha_{k} \frac{d^{k}}{d t^{k}}
$$

be a linear autonomous differential operator. Then, the stochastic differential equation $L X=Y$ has a continuous $\beta$-bounded solution if and only if it has a continuous $\beta$-bounded solution whose spectral measure is orthogonally scattered.

ACKNowledgement. The author would like to thank S. Cambanis, H. Hurd, G. Kallianpur and R. Leadbetter for their kind hospitality and support during his two year stay at The Center for Stochastic Processes. Thanks also to Michael Lacey whose suggestions helped to improve the presentation of this note.

\section{REFERENCES}

[B] S. Bochner, Stationarity, boundedness, almost periodicity of random valued functions, Proc. Third Berkeley Sympos. Math. Statist. Prob. 2 (1956), 7-27.

[C] H. Cramér, On harmonic analysis in certain functional spaces, Ark. Mat. Astr. Fys. 28B (1942), $7 \mathrm{pp}$.

[D] N. Dinculeanu, Vector measures, Pergamon Press, Oxford, 1967.

[GV] I. M. Gel'fand and N. Vilenkin, Generalized functions, Vol. 4, Applications of Harmonic Analysis, Academic Press, New York, 1964.

[Hi] T. Hida, Brownian motion, Applications of Mathematics, vol. 11, Springer-Verlag, Berlin and New York, 1980.

[Ho1] C. Houdré, Harmonizability, $V$-boundedness, (2,P)-boundedness of stochastic processes, Center for Stochastic Processes Tech. Rept. no. 239, Univ. of North Carolina, 1988, Prob. Theory and Rel. Fields (to appear).

[Ho2] _ Linear Fourier and stochastic analysis, Center for Stochastic Processes Tech. Rept. no. 258, University of North Carolina, 1989.

[K] I. Kluvánek, Characterization of Fourier-Stieltjes transformations of vector and operator valued measures, Czech. Math. J. 17 (1967), 261-277.

[N] H. Niemi, On orthogonality scattered dilations of bounded vector measures, Ann. Acad. Sci. Fenn. AI Math. 3 (1977), 43-52.

[Ph] R. S. Phillips, On Fourier-Stieltjes integrals, Trans. Amer. Math. Soc. 69 (1950), 312-323.

[Pi] G. Pisier, Factorization of linear operators and geometry of Banach spaces, CBMS Regional Conf. Ser. in Math. no. 60, Amer. Math. Soc., Providence, R.I., 1986.

[S] E. Slutsky, Sur les fonctions aléatoires presque périodiques et sur la décomposition des fonctions aléatoires stationnaires en composantes, Actualités. Sci. Ind. no. 738 (1938), 35-55, Hermann \& $C^{\text {ie }}$, Paris.

Center for Stochastic Processes, Department of Statistics, University of North Carolina, Chapel Hill, North Carolina 27599-3260

Current address: Center for Computational Statistics and Probability, George Mason University, Fairfax, Virginia 22030-4444 
